\title{
Liquid Flow-Through Cooling For Avionics Applications
}

\author{
M. Barwick, M. Midkiff, and D. Seals \\ AT\&T Bell Laboratories \\ Whippany, N.J. 07891
}

\begin{abstract}
An avionics cooling system has been developed that circulates fluid through the structural frame of its SEM-E modules. The liquid flow-through module frame is only 100 mils thick, yet is capable of maintaining low junction and surface temperatures for modules dissipating in excess of 200 watts. The module form/factor is compatible with existing SEM-E circuit board formats and connectors and features tool-less, dripless, quick-disconnect removal and insertion. A prototype avionics cooling system has been developed consisting of 52 liquid flow-through modules and a parallel feed rack/manifold. Both the module and the system have demonstrated outstanding cooling efficiency, and good reliability under preliminary shock and vibration testing.
\end{abstract}

Index Terms- Avionics, cooling, microelectronics, modules

\section{INTRODUCTION}

Thermal stress is a leading cause of electronic failure and its management threatens to become the number one problem of avionics system designers. The magnitude of this problem becomes apparent when one examines microelectronic trends. Microprocessor clock frequencies have been doubling every five years and are projected to continue to do so over the next ten years. Systems being deployed today typically have a 25 Mhz system clock. At this frequency, most CMOS devices dissipate between a quarter and two watts. Bipolar devices commonly exceed five watts, and some devices dissipate in excess of fifteen watts. As clock speeds increase, so does power dissipation. CMOS devices experience a linear increase in heat dissipation with clock speed; thus a $100 \mathrm{Mhz}$ device will dissipate four times the power dissipated at 25 Mhz.

As lithography shrinks the gate size, the power needed per gate decreases, but the number of gates per unit area increases at faster rate. We are simultaneously becoming quite adept at packaging microelectronics in smaller and smaller volumes. Most deployed systems have a ratio of active silicon to board area of about 3 percent. Some of the newer surface mount boards boast ratios as high as 15 percent. The introduction of multichip packaging and monolithic wafer scale technology will cause this ratio to exceed 30 per cent by the end of this century.

These trends indicate that 100 to 200 watt SEM-E modules will be commonplace by the year 2005 , and that thermal stress management will become a major problem. Higher thermal stresses translate into higher failure rates, shorter operational lifetimes, and higher logistics and maintenance costs. Thermally-related failures manifest themselves in a number of ways, ranging from thermal expansion mismatch problems such as solder joint failures to reduced component lifetimes. A typical 44 I/O leadless chip with a $2.5 \mathrm{ppm} /{ }^{\circ} \mathrm{C}$ thermal expansion mismatch will experience a 100 -fold increase in solder joint failures with a $30^{\circ} \mathrm{C}$ elevation in operating temperature. Thermal stresses can also crack delicate electronic components, delaminate boards, and accelerate moisture absorption and corrosion.

There are several ways of improving cooling efficiency. One way is to find a better medium to absorb and move heat energy. Another is to minimize the thermal resistivity between the heat source and the cooling medium. Both can be incorporated into a common cooling approach. Fluids are very good mediums for absorbing and moving heat. The difficult problem is minimizing thermal resistivity between the heat source and the fluid. Immersion cooling is the most straightforward way to accomplish this task, but presents a number of compatibility and maintainability problems for near-term application. Edge conduction liquid cooling eliminates these problems, but is limited by the inefficient thermal transfer path between the module frame, the cooling rail, and the heat transfer medium. Liquid flow-through (LFT) cooling offers a reasonable compromise since the fluid can be brought within a few millimeters of the heat source without the ensuing maintainability issues. LFT cooling is used to cool some supercomputers such as the CRAY YFP. To date, no known system has tool-less quick-disconnect modules, or is capable of functioning in the harsh avionics environment. The key to building a reliable liquid flowthrough system was the development of a thin, dripless, quick-disconnect SEM-E cooling frame.

\section{THE SEM-E COOLING FRAME DESIGN}

The design of our prototype liquid flow-through cooling frame was driven by four major requirements. First the frame had to be capable of adequately $\left(\mathrm{T}_{\mathrm{j}}<85^{\circ} \mathrm{C}\right)$ cooling a 200 watt module. This cooling had to be accomplished with flow rates and pressure drop comparable or lower than current edgeconduction designs. Secondly, frame format had to be compatible with existing SEM-E circuit boards and connector form/fit factors. The frame core had to be stable, yet thin enough to ensure the SEM stack height would be less 0.6 inches. Finally, the frame had to permit tool-less, dripless, quick-disconnect and insertion. 
The LFT prototype frame is a sandwich structure consisting of two 0.020 inch thick outer walls and a 0.060 inch thick finned core. The cone serves a dual purpose of heat exchanger and structural support member. The fin geometry determines the surface area, flow dynamics, and cooling efficiency. The frame looks like a conventional SEM-E module except for two quick disconnect (dripless) couplings (QDCs) integrated into the top, outer edge of the frame (See Figure 1). This outrigger design permits form/fit compatibility with most existing SEM-E circuit boards and card-edge connectors. This compatibility has been demonstrated with circuit boards and connectors from AT\& T's SEM-E production line.

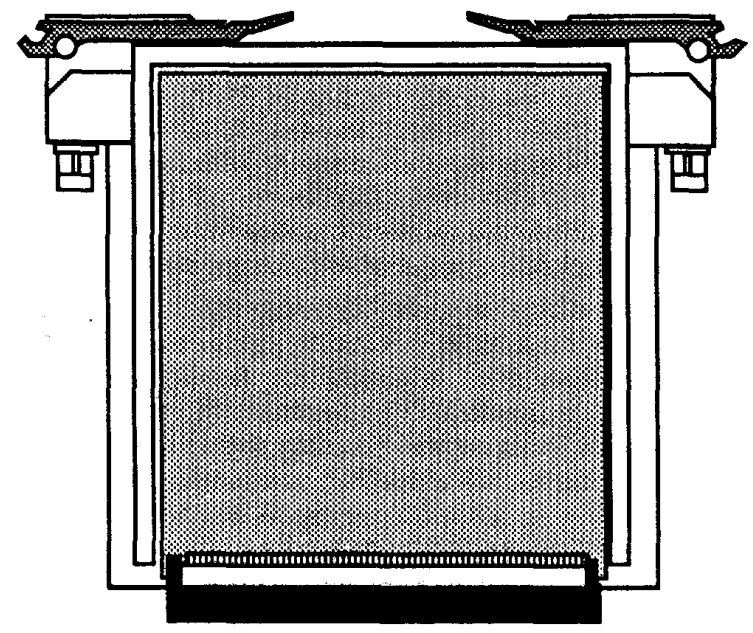

Figure 1 - The LFT SEM-E Module

A number of different LFT frames were fabricated to test and evaluate different design elements such as:

- Frame configuration,

- QDC connectors,

- Fin stock geometry, and

- Wedgelock design

The first set of frames differed only in the fin stock pattern and were used to select an optimal core design. Sixty additional frames (using this core design) were then fabricated for further thermal and mechanical testing.

\section{MODULE TEST RESULTS}

The first tests focused on establishing an empirical relationship between fin geometry, heat removal, pressure drop, and flow rates. Seven different fin geometries were evaluated, differing in fin length, width, and flow patterns. Each different frame was instrumented with 25 thermocouples and patch heaters. Considerable care was taken to eliminate any secondary cooling effects and ensure that all heat was removed via the cooling fluid. The frame was wrapped in insulation to eliminate any external conduction and radiation effects, while no contact was allowed with the rib rails to eliminate edge conduction effects. These precautions were necessary to ensure the accuracy of cooling efficiency measurements.

The first series of tests were conducted on a 100 watt module using a polyalphaolefin (Brayco Micronic 889 ) as a coolant. Flow rates between 0.05 and $0.1 \mathrm{gpm}$ were sufficient to restrict frame temperatures to less than a $20^{\circ} \mathrm{C}$ rise over the inlet coolant temperature. Pressure drops ranged between 0.5 and $6 \mathrm{psi}$. Further tests showed that the better core designs were capable maintaining a $25^{\circ} \mathrm{C}$ frame tempcrature rise for a 200 watt SEM-E module with only modest flow rates and pressure drops. This rise is much less than the thermal resistivity across wedgelock/rail interface of the edge-conduction card alone! Fluids with specific heats greater than polyalpholefins could be used for modules dissipating in excess of 200 watts. Water could cool modules dissipating up to 500 watts at the same flow rates.

Flow tests showed that most of the pressure drop (75\% to $99 \%$ ) occurs across the fluid connectors. This indicates that a thinner (perhaps as thin as 0.060 inches) core could be used without incurring significantly larger pressure drops. New fluid fittings (Snaptite, Hydraflow) have already been developed that reduce connector pressure drop by another factor of two.

Several burst pressure tests were conducted on the bare frames and the burst pressures were well in excess of the projected worst case operating pressure of $50 \mathrm{psi}$.

Module level mechanical (shock, vibration) testing was conducted on two prototypes. Both modules comprised a corc 7 LFT frame and 4:1 extraction levers, but differed in the choice of card guide retention and MIB design. The first module was a tool-less removable module fitted with leveractuated wedge locks designed by Lockhart Industries. Ceramic MIBs taken from one of AT\&T's production SEMEs (no devices soldered to MIBs) were attached to both sides of the frame. The second module was outfitted with torqueactuated Birtcher wedge locks and two special MIBs. One MIB was a production unit rejected for excessive bowing, and the other was designed for solder joint reliability testing.

A $5 \mathrm{~g}$ sine resonance search was performed along each of the three axes of the lever-actuated module. A twenty minute sweep from 10 to $2000 \mathrm{~Hz}$ was conducted along each axis. Six accelerometers provided surface displacement monitoring during the testing. Brayco 889 Micronic coolant $\left(25^{\circ} \mathrm{C}\right)$ was circulated through the module during the testing. The results of these tests showed the $\mathrm{z}$-axis (module bending like a thin plate) to be the primary direction of motion, therefore tests on the torque-actuated module were restricted to the $\mathrm{z}$-axis.

The lever-actuated module exhibited its first natural harmonic at $678 \mathrm{~Hz}$. The point of maximum deflection was found to be centered at the top edge of the module between the two QDC outriggers. The torque-actuated module (12 inch-pounds of torque) was subjected to the same $5 \mathrm{~g}$ sine sweep. Its natural frequency $\left(f_{n}\right)$ occurred at $792 \mathrm{~Hz}$ and with a similar maximum displacement location. The increase in $\mathrm{f}_{\mathrm{n}}$ is due to the greater fixity provided by the lever-actuated wedge locks. 
Additional sine sweep tests were conducted on the torqueactuated module to determine if its natural frequency might vary (1) with complete fluid loss, (2) elevated fluid temperature (restricted flow or loss of flow), and (3) lower torque down forces. Loss of fluid was found to have no impact on the module's first natural frequency $(792 \mathrm{~Hz})$, but as the fluid temperature approached the glass transition temperature $\left(55^{\circ} \mathrm{C}\right)$ of the circuit board adhesive, the natural frequency dropped to $725 \mathrm{~Hz}$. The torque down force had the greatest impact on the natural frequency, as 8 inch-pound torque lowered $f_{n}$ to $713 \mathrm{~Hz}$. The maximum double amplitude displacements ranged from 0.002 to 0.004 inches for the various module configurations.

Random vibration tests were conducted on the leveractuated module using the following test profile:

- 50 - $100 \mathrm{~Hz}: 6 \mathrm{db} /$ octave

- $100-1000 \mathrm{~Hz}: 0.2 \mathrm{~g}^{2} / \mathrm{Hz}$

- 1000 - $2000 \mathrm{~Hz}:-6 \mathrm{db} /$ octave.

The input rms g level was $16.91 \mathrm{~g} \mathrm{~s}$. Tests were conducted in the $\mathrm{x}$ and $\mathrm{y}$ axes for $1 / 2$ hour with fluid flow and electrical fault monitor. Four electrical nets in the MIB were monitored continuously for intermittent signal interruption. In addition, 20 other nets were checked every hour for permanent loss of signal. . Since the $\mathrm{z}$-axis showed the greatest displacement, $\mathrm{z}$-axis testing was performed for a total of 6 hours.

Examination of the response plot for the front/center of the lever-actuated module showed an $\mathrm{f}_{n}$ shift from $678 \mathrm{~Hz}$ in sine to $565 \mathrm{~Hz}$ in random vibration. This shift is larger than that observed in our production solid core modules (they exhibit a shift of $1100 \mathrm{~Hz}$ to $1055 \mathrm{~Hz}$ ). The unusual configuration of the LFT module, coupled with the use of the lower fixity wedge locks, reduced core thickness, and increased weight are believed to be the cause of this shift.

The transmissibility was measured to 10 at the $565 \mathrm{~Hz}$ resonance, and a 56.6 output $\mathrm{mms} \mathrm{g}$ level. Assuming that the module behaves as a single degree of freedom system, the number of random vibration stress reversals after 6 hours of testing was calculated to be:

- $8.3 \times 10^{6}$ reversals of 0.0017 inches,

- $3.3 \times 10^{6}$ reversals of 0.0034 inches, and

- $0.5 \times 106$ reversals of 0.0052 inches.

There were no electrical failures during the testing. No leakage was observed, nor was there any loss of function of the QDCs or the lever-actuated wedges.

Subsequent random vibration testing was conducted in an attempt to accelerate the module to failure. A ramped random vibration test was started at the initial test levels and the power spectral density was increased by $5 \mathrm{db}$ every 10 minutes. Near the conclusion of the tests, module was driven at a $37.8 \mathrm{rms} \mathrm{g}$ input level with a one sigma response of 145 rms gs and 3 sigma displacements in excess of .020 inches. The were no electrical or mechanical failures observed. The module was calculated to have undergone an additional 5 million stress reversals. No mechanical or electrical failure were found during or after the testing.

\section{THE RACK/MANIFOLD DESIGN}

The rack was designed (See Figure 2) so that all modules have equal access to the coolant. Fluid flows into each supply manifold and is distributed to the inlet fluid connector of each module. The coolant exits the module and flows into a return manifold. This parallel flow design ensures that module temperatures, flow rates, and pressure drops are relatively unaffected by the number and distribution of modules in the rack. Special header plates permit multiple bay designs, each with their own supply and return columns.

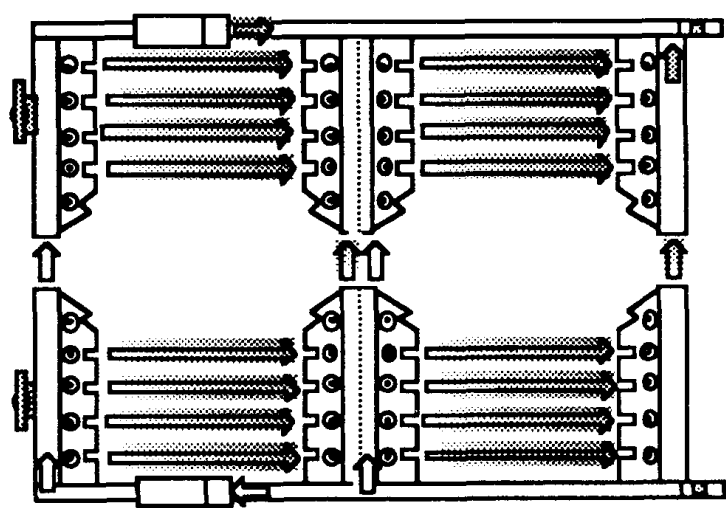

Figure 2 - Rack Flow Distribution

System level testing was conducted on a two bay rack and backplane that spanned both bays. The rack was hinged to permit backplane access during maintenance. One bay was populated with 26 torque-actuated module, while the other bay held 26 lever-actuated modules. Five modules in each bay were fitted with a new low resistance QDC obtained just prior to testing. This test unit was attached to the electrodynamic shake table using a special fixture designed to duplicate the fixity of an airbome enclosure.

\section{SYSTEM TEST RESULTS} tests:

The prototype system was subjected to the following

- Mechanical shock,

- Sinusoidal vibration,

- Random vibration, and

- Thermal efficiency measurements.

All tests were conducted with with Brayco 889 Micronic as the coolant with flow rates of $4 \mathrm{gpm}$. Thermal tests were conducted with an average module power dissipation of 50 watts.

The mechanical shock tests consisted of 18 saw-tooth pulse shocks of 11 millisecond duration and $20 \mathrm{~g}$ magnitude. 
The shocks were directed along each of the 3 rack axes. No leaks or loss of mechanical integrity was observed.

A $0.25 \mathrm{~g}$ sine resonance search was performed from 10 to $2000 \mathrm{~Hz}$ in 8 minute sweeps for each axis. Preliminary analysis of the test results indicates the first rack resonance to occur at $278 \mathrm{~Hz}$, while the first module resonance occurs at $794 \mathrm{~Hz}$ (as measured in the rack/manifold assembly). Since the module resonance is more than double the rack resonance, they are believed to be sufficiently decoupled for avionics applications.

The random vibration profile was derived from data for the F-15 aircraft. The $x, y$ axes were excited for $1 / 2$ hour intervals. Since the module z-axis exhibited the greatest displacements, it was tested for 5.5 hours. No other loss of mechanical function was encountered.

During the course of the mechanical testing, periodic extraction/insertion tests were conducted and the discharge side of several QDC nipple assemblies were found to be jammed in the open position. This problem had no effect on the QDC's ability to seal while engaged, but leakage would occur upon disengagement. Furthermore, if the disengagement occurred while the system was pressurized, the coolant would escape in an atomized mist. The cause of this problem has been traced to small pieces of aluminum left by the QDC port thread tapping operation. All failures occurred in the discharge side of the QDC (indicating that the choke point is unidirectional), and only occurred in the QDCs with a narrow gland design.

Although careful cleaning and quality control during fabrication should eliminate this problem, several additional steps were taken in the design to ensure against the reoccurrence of this problem. A second generation of QDCs with larger glands are being incorporated in the design. Several of these new QDCs were included in our test unit, and no blockages were observed. These new QDCs also provide the additional benefits of lower pressure drops Filter screens are also being incorporated as part of the design improvement.

Wearout analysis of the LFT system after 12 million stress reversal cycles show the O-rings at the interface between the QDC coupler and nipple assembly halves to exhibit the greatest wear. Although no leaks were attributable to O-ring wearout, further study is needed to insure that a combination of vibration and other stressor will not precipitate pre-mature wearout.

\section{CONCLUSIONS}

LFT cooling tests show a significant improvement over edge-conduction cooling. Thermal comparison tests indicate a $20^{\circ} \mathrm{C}$ improvement in frame surface temperature over an edge-conduction cooled 50 watt module. Since cooling benefits will increases linearly with module power dissipation, this improvement is projected to rise to $40^{\circ} \mathrm{C}$ for a 100 watt module.

The results of the mechanical testing show that a LFT could meet avionics shock and vibration requirements with only minor improvements made to the original prototype design. Leakage and storage of LFT modules do not appear to be a major problem, although further testing is needed to confirm this assertion.

Logistics and maintenance of the modules should be similar to conventional solid frame SEM-Es. The leveractuated wedge locks supplied by Lockhart Industries have provided adequate retention throughout our testing and no failures were observed. Insertion and removal of the leveractuated modules was significantly faster and easier than the torqued version.

The LFT rack/manifold is also capable of edge conduction cooling, although at a slightly lower efficiency than an optimized edge-conduction rack/manifold This opens up the possibility of mixing LFT and edge-conduction modules in the same rack/manifold. This feature, along with the relative ease of migrating existing circuit cards and connectors to the LFT frame will aid the smooth migration of LFT systems into the inventory. Further studies are needed into system weight, overall module reliability, and life cycle cost to determine the proper course of integration.

\section{ACKNOWLEDGEMENTS}

The authors wish to acknowledge the contribution of the Wright Research Development Center and the AAAPT program management. Much of the research and material in this paper was funded under their program. We are particularly indebted to Marvin Soraya, John Ostgaard, and Reed Morgan for their many helpful comments and suggestions. 